Artículo Original

\title{
Descripción de una nueva especie de Salpingus Illiger (Coleoptera: Salpingidae) para Chile
}

Description of a new species of Salpingus Illiger (Coleoptera: Salpingidae) from Chile

Jaime Solervicens A. ${ }^{1}$

${ }^{1}$ Sociedad Chilena de Entomología, Santiago, Chile.E-mail: jaime.solervicens@umce.cl

\section{ZooBank: urn:lsid:zoobank.org:pub: 407F02A8-33C9-485C-980F-47483ABC258D https: / / doi.org/10.35249/ rche.46.3.20.04}

Resumen. Se describe Salpingus rucamanque sp. nov., que se adscribe tentativamente al género Salpingus Illiger. Se presentan imágenes de su aspecto externo y se dan datos de su distribución. Se establecen las diferencias de la nueva especie con las previamente descritas en Rhinosimus (hoy Salpingus) para Chile.

Palabras clave: Salpinginae, Salpingini, subregión subantártica.

Abstract. Salpingus rucamanque nov. sp. is described and tentatively ascribed to the genus Salpingus Illiger. Images of its external appearance are presented and data on its distribution are given. The differences of the new species with those previously described in Rhinosimus (now Salpingus) from Chile are established.

Key words: Salpinginae, Salpingini, subantarctic subregión.

\section{Introducción}

La mayoría de las especies de Salpingidae chilenos se ubican en la actual subfamilia Salpinginae, siete de ellas en la tribu Salpingini y una en Lissodemini. Entre los Salpingini, cuatro especies fueron descritas originalmente en el género Salpingus Illiger, 1802: $S$. variegatus y $S$. anthracinus por Fairmaire y Germain (1861) y S. andinus y $S$. frigidus por Fairmaire y Germain (1863); dos en Rhinosimus Latreille, 1802: R. brevirostris Fairmaire y Germain, 1863 y R. valdivianus Philippi y Philippi, 1864 y una en Oncosalpingus Blair, 1919: O. podagricus Blair, 1919 (Fairmaire y Germain 1861, 1863; Philippi y Philippi 1864; Blair 1919; Blackwelder 1945; Elgueta y Arriagada 1989). S. variegatus, S. andinus y S. frigidus, así como R. brevirostris, fueron transferidos a Vincenzellus Reitter, 1911 (Blackwelder 1945; Elgueta y Arriagada 1989). S. anthracinus fue ubicado por sus propios autores en Rhinosimus (Fairmaire y Germain 1863) y más tarde clasificado en el género Cariderus Mulsant, 1859 (Blackwelder 1945). R. valdivianus está siendo transferido a un género nuevo dentro de la tribu Lissodemini (Solervicens 2020), en tanto Oncosalpingus persiste como entidad válida. Recientemente, Rhinosimus ha sido reconocido como sinónimo de Salpingus Illiger, 1802 (Pollock y Löbl 2008; Lawrence et al. 2010b).

Recolecciones recientes han proporcionado ejemplares de una nueva especie que se

Recibido 11 Junio 2020 / Aceptado 8 Julio 2020 / Publicado online 24 Julio 2020

Editor Responsable: José Mondaca E. 
atribuye tentativamente al género Salpingus Illiger, la cual se describe a continuación.

\section{Materiales y Métodos}

La descripción de la nueva especie se basó en cuatro individuos pertenecientes a la colección entomológica del Museo Nacional de Historia Natural de Santiago, Chile (MNNC), conservados aparte como especies relevantes para estudio. Las medidas del cuerpo de los cuatro ejemplares estudiados se tomaron bajo estereomicroscopio mediante reglilla graduada; ya sea que se trate de un valor promedio o de relaciones de medidas siempre se indica su rango. El largo del cuerpo se consideró entre el borde anterior de la cabeza en posición flectada ortoñata (por lo que no incluye el rostro) y el ápice de los élitros; el rostro se midió entre el borde anterior de los ojos y el borde distal del labro. Las fotos se tomaron con una cámara digital Panasonic LX3 a través del ocular de un estereomicroscopio Olympus SZ51. La terminología empleada en la descripción sigue a la propuesta por Lawrence et al. (2010a).

\section{Resultados}

\section{Salpingus rucamanque sp. nov.}

(Figs. 1-3)

Descripción. Largo promedio del cuerpo: 2,59 mm (rango: 2,50-2,65), 2,5 veces más largo que ancho (rango: 2,38-2,63) (Fig. 1). Cabeza: Ancho a nivel de los ojos menor que el ancho máximo del pronoto (0,86:1, rango: 0,84-0,89); frente subplana, puede presentar un pequeño promontorio en la base del rostro; vértex convexo; temples cortos, 0,42 veces el largo antero-posterior del ojo (rango: 0,33-0,54); rostro (Fig. 2) 1,77 veces más largo que la cabeza (rango: 1,72-1,81) y poco más largo que el ancho de esta $(1,08: 1$, rango: 1,00-1,13), fuertemente comprimido dorso-ventralmente, medianamente curvado ventralmente, lados convergentes en su base, paralelos en el tramo medio y divergentes en el ápice, orientado perpendicularmente al cuerpo en los ejemplares montados (condición que se modificó en el ejemplar preparado para fotografía); ojos laterales, hemisféricos, enteros, sobresalientes, con facetas finas; antenas largas, orientadas hacia atrás sobrepasan un poco los ángulos humerales, insertadas un poco por delante de los ojos, el espacio entre la inserción de la antena y el borde anterior del ojo aproximadamente como el ancho del escapo, escapo oblongo, pedicelo y segmentos 3 a 6 filiformes, levemente dilatados hacia el ápice, segmentos 7 a 11 ensanchados formando maza, con pilosidad corta, densa, 7 a 10 cónicos, 11 oblongo, todos los segmentos de la antena con algunos pelos largos, oblicuos; mandíbulas bidentadas en el ápice; palpos maxilares con tres segmentos expuestos en el extremo del rostro, el último más grande, oblongo; palpos labiales pequeños, no sobresalientes; gula de lados rectos, convergentes hacia adelante. Superficie brillante, puntuación fuerte y densa, puntos corrientemente separados por un espacio menor a su diámetro, más espaciados a ausentes en el promontorio frontal y en la parte media del vértex; rostro liso y brillante en su parte media y con puntos angostos y largos en los costados. Pilosidad blanquecina rala, erguida e inclinada. Tórax: Pronoto (Fig. 3) levemente más ancho que largo (1,07:1, rango: 1,04-1,12), ancho anterior igual o ligeramente menor que el posterior (0,98:1, rango: 0,95-1,00), ancho máximo en tercio anterior, esta amplitud menor que el ancho humeral (0,72:1, rango: 0,68-0,75); costados mayoritariamente convexos, subrectos en la base, sin reborde lateral; borde anterior y posterior finamente carenados; disco convexo transversalmente, en el tercio anterior con una depresión media y dos depresiones laterales menos acentuadas, impresiones prebasales transversas, bien marcadas. Puntuación más fuerte que en la cabeza, densa, 
compromete también a los hipómeros. Pilosidad moderada a base de pelos blanquecinos erguidos e inclinados, que a cada lado de la línea media se orientan hacia atrás. Prosternón delante de las coxas poco más largo que el diámetro antero-posterior de una de ellas (12/10), sutura notoesternal marcada, cavidades coxales contiguas, abiertas. Mesoventrito poco más largo como el diámetro antero-posterior de su coxa (15/13), sutura con el mesanepisterno débil, mesoepímero triangular, proceso mesoventral angosto, cavidades coxales subcontiguas, cerradas. Metaventrito amplio, convexo, parte central en dos tercios posteriores aplanada formando dos franjas lisas y brillantes, separadas por una fila de puntos; parte lateral posterior algo abombada, con un área lisa marcada con puntos aislados o sin ellos, ubicada junto a la coxa; sutura metakatepisternal marcada, espacio intercoxal posterior apenas escotado; metanepisterno alargado, poco angostado hacia atrás; metaepímero visible. Región ventral de los segmentos torácicos con puntuación fuerte, densa, homogénea, como en el pronoto y pilosidad a base de pelos blanquecinos inclinados, ralos. Escutelo triangular, liso (Fig. 3). Élitros: Aproximadamente dos veces más largos que el ancho humeral (2,08:1, rango: 2,04-2,12) y poco más de tres veces el largo del pronoto (3,15:1, rango: $2,95-3,33)$, su mayor amplitud en la mitad $(1,14: 1$ con respecto a la base, rango: 1,08-1,20); disco convexo en sentido transversal, área basal abombada, limitada atrás por leve depresión curva entre la sutura y el ángulo humeral, superficie recorrida por once filas de puntos grandes como en el pronoto que reducen su tamaño hacia el ápice, espacios entre las filas lisos y sin puntos, ángulo posterior interno cortamente redondeado; pilosidad destacada, relativamente densa, constituida por pelos blanquecinos largos, inclinados hacia atrás. Epipleura angosta, lisa, con algunos puntos menores, se angosta hacia atrás y desaparece en la curvatura posterior del ala. Alas metatorácicas bien desarrolladas. Patas: Coxas anteriores cónicas, coxas medias globosas; unión trocántero-femoral oblicua; fémures fusiformes fuertemente angostados hacia la base; tibias delgadas, poco ensanchadas hacia el ápice, con dos espolones; tarsos 5-5-4, segmentos primero al penúltimo densamente pilosos por ventral, donde el primero es el más largo y el penúltimo el más corto, inserción de cada segmento en escotadura dorsal del precedente; garras simples. Abdomen: Con cinco ventritos, gradualmente más cortos del 1 al 5, borde posterior recto, costados con fino reborde, convexos en sentido transversal, primer ventrito con proceso intercoxal agudo; puntuación densa, homogénea, puntos algo menores que en la parte ventral del tórax; pilosidad más o menos abundante, a base de pelos finos, blanquecinos, inclinados. Pigidio de costados convergentes hacia atrás, borde posterior convexo a subrecto, ángulos redondeados, disco moderadamente convexo. Coloración: Cabeza pardo rojizo oscuro, rostro pardo rojizo; antenas completamente pardas o con segmentos basales pardo rojizo; pronoto, partes ventrales del tórax, abdomen y patas pardo rojizo, tibias y tarsos pueden ser más claros; escutelo pardo oscuro; élitros pardos a pardo amarillentos El color de la cabeza contrasta con el del resto del cuerpo.

Etimología. Especie nombrada de acuerdo con su localidad de recolección.

Distribución. Los únicos registros de esta especie provienen del Parque Ecológico y Cultural Rucamanque de la Universidad de la Frontera, ubicado al noroeste de la ciudad de Temuco, Provincia de Cautín, Región de La Araucanía, cuya vegetación corresponde a la sub-región del Bosque Caducifolio del Llano (Gajardo 1994). Los ejemplares fueron capturados sobre quila (Chusquea sp.) en el borde del bosque.

Material tipo. HOLOTIPO, hembra: Chile, Cautín, Rucamanque, en quila, borde bosque, Nov. 2007, A. Fierro, Salpingus rucamanque J. Solervicens det. 2020 (MNNC). PARATIPOS: 3 ejemplares, sexo no determinado, con los mismos datos del holotipo (MNNC). 


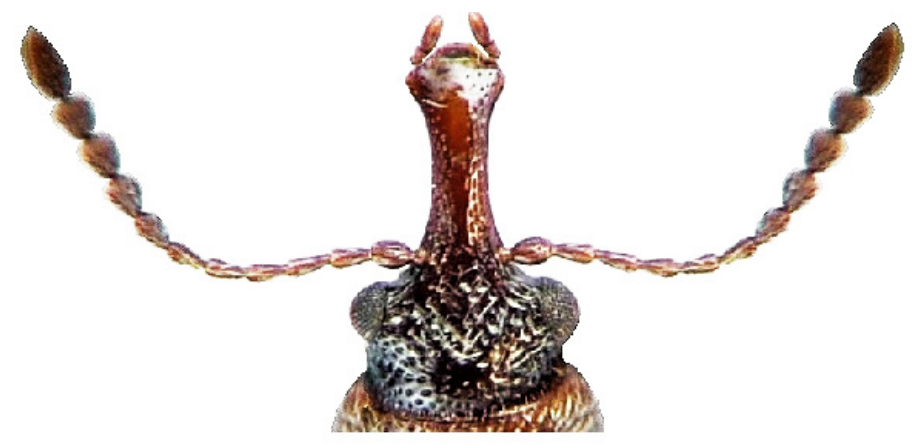

2
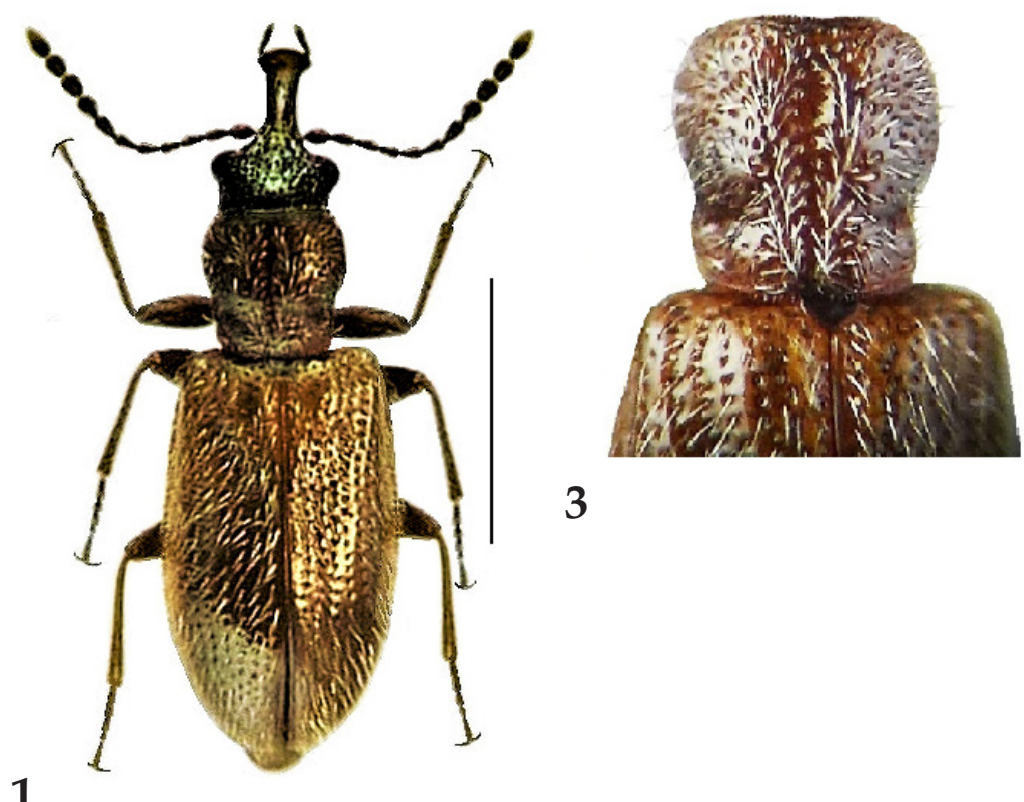

3

Figuras 1-3. Salpingus rucamanque sp. nov. 1. Habitus dorsal (Escala: $1 \mathrm{~mm})$. 2. Detalle de la cabeza, rostro y antenas. 3. Pronoto y escutelo.

\section{Discusión}

La mayoría de las especies de Salpinginae del hemisferio sur han sido clasificadas en taxones holárticos y se estima que probablemente requieran ser ubicadas en nuevos géneros cuando se haga un estudio cladístico amplio (Pollock 2002; Lawrence et al. 2010b). Este es el caso de la mayor parte de los salpínginos chilenos (Blackwelder 1945; Elgueta y Arriagada 1989). La especie que se describe en este trabajo había sido inicialmente atribuida a Rhinosimus, pero la estabilidad de este género está cuestionada pues varias de sus especies han sido clasificadas en los géneros Cariderus Mulsant, 1859, Salpingus Illiger, 1802 y Vincenzellus Reitter, 1811 y, más aún, su especie tipo ha sido reconocida en la sinonimia de Salpingus Illiger (Pollock y Löbl 2008; Lawrence et al. 2010b). Sobre la base de estos antecedentes el nuevo taxón se ha clasificado tentativamente en Salpingus, pero su posición definitiva queda a la espera de análisis filogenéticos de los taxa.

En relación con las especies chilenas ubicadas anteriormente en Rhinosimus (Elgueta 
y Arriagada 1989), se debe descartar a $R$. valdivianus, pues la maza antenal de tres segmentos y la presencia de espinas en los costados del pronoto permiten reconocerla como perteneciente a un género distinto atribuido a la tribu Lissodemini (Solervicens 2020). Al comparar R. anthracinus (basado en su descripción), hoy Salpingus anthracinus, con S. rucamanque, es posible detectar diferencias entre ambas (ver Tabla 1) suficientemente marcadas que aseguran la validez de la nueva especie.

Tabla 1. Caracteres diferenciales entre S. anthracinus y S. rucamanque sp. nov.

\begin{tabular}{|l|l|l|}
\hline Caracteres & Salpingus anthracinus & Salpingus rucamanque \\
\hline Largo del cuerpo & $3,75 \mathrm{~mm}$ & $2,6 \mathrm{~mm}$ \\
\hline Puntuación cabeza y pronoto & oblonga & redondeada \\
\hline Inserción antenas en el rostro & antes de la mitad & en la base \\
\hline Base élitros & No abombada & abombada \\
\hline Coloración élitros & con manchas & uniforme \\
\hline
\end{tabular}

\section{Agradecimientos}

A Mario Elgueta D. curador de la colección entomológica del Museo Nacional de Historia Natural de Santiago, Chile, por facilitar el material objeto de esta contribución y por sus comentarios al manuscrito que mejoraron sustancialmente sus resultados. A Andrés Fierro, recolector de los ejemplares estudiados, por ceder generosamente dicho material al Museo Nacional de Historia Natural de Chile.

\section{Literatura Citada}

Blackwelder, R.E. (1945) Checklist of the Coleopterous Insects of México, Central America, the West Indies, and South America. Bulletin United States National Museum, 185(3): 343-550.

Elgueta, M. y Arriagada, G. (1989) Estado actual del conocimiento de los coleópteros de Chile (Insecta: Coleoptera). Revista Chilena de Entomología, 17: 5-60.

Fairmaire, L. y Germain, P. (1861) Coleoptera Chilensia, París, 8 pp.

Fairmaire, L. y Germain, P. (1863) Révision des coléopteres du Chili. Annales de la Société Entomologique de France, série 4, 3: 225-284.

Gajardo, R. (1994) La vegetación natural de Chile. Clasificación y distribución geográfica. Editorial Universitaria, Santiago, Chile, 165 pp.

Lawrence, J.F., Beutel, R.G., Leschen, R.A.B. y Ślipiński, A. (2010a) Glossary of morphological terms, pp. 9-20. En: Leschen, R. A. B., Beutel, R. G. \& Lawrence, J. F. (eds.), Handbook of Zoology, Coleoptera, Beetles, Vol. 2: Morphology and Systematics (Elateroidea, Bostrichiformia, Cucujiformia). Walter de Gruyter GmbH \& Co., Berlín, New York, 785 pp.

Lawrence, J.F., Ślipiński, A., Pollock D.A. y Escalona, H. (2010b) Salpingidae Leach, 1815, pp. 722-729. En: Leschen, R.A.B., Beutel, R.G. y Lawrence, J.F. (eds.) Handbook of Zoology, Coleoptera, Beetles, Vol. 2: Morphology and Systematics (Elateroidea, Bostrichiformia, Cucujiformiapartim). Walter de Gruyter GmbH \& Co., Berlín, New York, 785 pp.

Philippi, R.A. y Philippi, F. (1864) Beschreibung einiger neuen Chilenischen Käfer. Entomologische Zeitung, 25: 313-406.

Pollock, D.A. (2002) 116 Salpingidae Leach, 1815. Pp. 544-548. En: Arnett, R. H. Jr., Thomas, M.C. Skelley, P. E. y Frank, J. H. (eds.) American Beetles, Volumen 2. Polyphaga: Scarabaeoidea through Curculionoidea. CRC Press, Gainesville, 861 pp. 
Pollock, D.A. y Löbl, I. (2008) Family Salpingidae Leach,1815, pp. 417-421. En: Löbl, I. \& Smetana, A.(eds.): Catalogue of Palaearctic Coleoptera, vol. 5. Tenebrionoidea. Apollo Books Stenstrup, 670 pp.

Solervicens, J. (2020) Rhinolissomus, nuevo género para Rhinosimus valdivianus Philippi y Philippi, 1864 (Coleoptera: Salpingidae). Revista Chilena de Entomología, 46(3): 373-379. 